\title{
Neue Wege in der Finanzierung der deutschen Krankenhäuser 2020 - Der "Pflexit" und das neue aG-DRG-System
}

Mit der Ausgliederung der Pflegepersonalkosten aus der fallpauschalierenden Vergütung und der Einführung der nahezu vollständigen Selbstkostendeckung im Bereich der „Pflege am Bett“ wird ein Paradigmenwechsel vollzogen. Durch die parallele Nutzung zweier Abrechnungssysteme, der Notwendigkeit einer nicht gerade simplen $\mathrm{Ab}$ grenzung der vom Pflegebudget umfassten Pflegekosten und der vielfältigen Themen, die 2020 jedes Krankenhaus mit den Kostenträgern (neu) vereinbaren muss, ist der Komplexitätsgrad in der Krankenhausfinanzierung in einem schwer nachvollziehbaren Ausmaß gestiegen. Während der „Pflexit“ auf die Kodierung zunächst keinen Einfluss nimmt, kann er die zukünftigen Erlöse einzelner Krankenhäuser erheblich beeinflussen. Neben den in das Pflegebudget eingehenden Kosten bestimmt die (Methodik der) Ausgliederung der Pflegepersonalkosten aus den G-DRGs auch nachhaltig die Erlöse, die über die Rumpf-DRGs (,aG-DRGs“) generiert werden. Die Einflüsse der jährlichen Revision des G-DRG-Systems treten damit für 2020 für viele Krankenhäuser in den Hintergrund.

Der Blick auf den aG-DRG-Fallpauschalenkatalog 2020 und die tagesbezogenen Pflegeentgelte offenbart, dass aus den typisch rheumatologischen G-DRGs sehr wenig Pflegepersonalkosten ausgegliedert wurden. Die rheumatologischen G-DRGs I66E, I66G, I69A, I79Z und I97Z gehören zu den 12 G-DRGs (1\%) mit den niedrigsten tagesbezogenen Pflegeerlösen aller 1272 bewerteten vollstationären DRGs. Die G-DRG I79Z (Fibromyalgie) weist dabei sogar die drittniedrigste und die G-DRG $197 Z$ (Rheumatologische Komplexbehandlung) die viertniedrigste tagesbezogene Pflege-Bewertungsrelation aller vollstationären DRGs auf. Entsprechend gehen nicht so viele Erlöse durch die Ausgliederung der Pflegepersonalkosten verloren wie bei anderen G-DRGs.
Rheumatologische Krankenhäuser, die demgegenüber hohe Pflegepersonalkosten für das Pflegebudget gelten machen können, z. B. über Personalaufbau in der Pflege und Abbau anderer Berufsgruppen, dürften zunächst zu den Gewinnern gehören. Zusätzlich dürfen Krankenhäuser (außer in Rheinland-Pfalz) im nächsten Jahr auch von einer ungewöhnlichen hohen Steigerung der Preiskomponente (Landesbasisfallwerte) von über $3 \%$ und weiteren - teilweise für 2020 limitierten - Aufschlägen profitieren. Nicht zu verachten ist jedoch, dass sich die Kalkulationsbasis für die rheumatologischen G-DRG geändert, d. h. auch erweitert haben dürfte. Damit scheinen Leistungserbringer mit günstigerer Kostenstruktur die Kalkulation einzelner rheumatologischer DRGs beeinflusst zu haben. So wurde die G-DRG I97Z (Rheumatologische Komplexbehandlung) anhand von 3559 Fällen (Vorjahr 2780 Fälle; + $28 \%$ ) und die G-DRG I79Z (Fibromyalgie) anhand von 2125 Fällen (Vorjahr 1715 Fälle; +24\%) kalkuliert. Werden die Bewertungsrelationen (ohne Pflege) zwischen 2019 und 2020 verglichen, so sind diese für die G-DRG $197 Z$ (Rheumatologische Komplexbehandlung) um -6,15\% und für die G-DRG I79Z (Fibromyalgie) um $-10,4 \%$ gesunken. Während der Kalkulation der G-DRG I79Z auch Fälle mit einer im Mittel 0,8 Tage kürzeren Verweildauer zugrunde lagen, stieg die mittlere Verweildauer für die G-DRG I97Z sogar leichtgradig (0,1 Tage, obere Grenzverweildauer + 1 Tag) an. Nach Ausgliederung der Pflegepersonalkosten stellen in beiden G-DRGs die Infrastrukturkosten mehr als die Hälfte der Kosten in der Kalkulation dar. Insgesamt wird diese Grenze nun bei 19 G-DRGs überschritten. Krankenhäuser, die sich auf diese Leistungen spezialisiert haben, dürften Budgetanteile verlieren.

Anders dürfte dies sich für rheumatologische Kliniken darstellen, die ihre Hauptleis- tungen im Bereich der Basis-DRGs 166 und 169 erbringen. Auf Antrag des VRA wurde die im Vorjahr durchgeführte Zusammenfassung der G-DRGs I69A und I69B zu einer gemeinsamen G-DRG I69Z vom InEK wieder zurückgenommen. In der G-DRG I69B werden nun nur noch Arthrosen abgebildet. Alle weiteren Hauptdiagnosen - insbesondere die entzündlich-rheumatischen Erkrankungen - kommen wieder in die höher bewertete G-DRG I69A. Die Bewertungsrelation liegt hier 3,7\% höher als die der G-DRG I69Z im Vorjahr (die I69B mit den Arthrosen wurde hingegen um -13,8\% abgewertet). Auch die G-DRG I66G erfuhr eine geringe Aufwertung von 0,9\%. Auch in den G-DRGs I69A und I66G liegt der Anteil der Infrastrukturkosten über $45 \%$. Im Kontext der Vielzahl der Veränderungen, muss daher jede rheumatologische Klinik selbst ermitteln, ob sie 2020 zu den Gewinnern oder Verlieren gehört. Dabei sollten auch die Neuerungen des MDK-Reformgesetzes mit beachtet werden.

\section{Einzelfallprüfungen, Prüfquoten und Strafzahlungen nach dem MDK-Reformgesetz}

Aufgrund stetig steigender Prüfquoten und dem Druck des Bundesrechnungshofs hat sich der Gesetzgeber nach langer Zeit wieder an eine Veränderung der Einzelfallprüfungen der Krankenhausabrechnungen durch die Krankenkassen getraut. Die initialen Hoffnungen der Krankenhäuser auf eine bürokratische und ggf. sogar finanzielle Entlastung dürften spätestens durch die sprichwörtlich in letzter Minute in das Gesetz aufgenommenen Änderungen weitestgehend zerstört worden sein. Zwar wird ab 2020 die Zahl der vollstationären Fälle, die die Krankenkassen mit Hilfe des MD (ehemals MDK) prüfen lassen dürfen, beschränkt (2020: 12,5 \%, 2021 Prüfquote quartalsbezogen 
abhängig von der Quote gekürzter Rechnungen), allerdings werden empfindliche Strafzahlungen fällig, wenn Kürzungen erfolgen. 2020 liegen diese noch bei $10 \%$ des Differenzbetrags (minimal allerdings $300 €$ ), ab 2021 ergibt sich die Höhe der Strafzahlung nach einem komplexen System, liegt aber ebenfalls minimal bei $300 €$. Die quartalsbezogenen Prüfquoten und Strafzahlungen werden vermutlich einen wechselnden Einfluss auf die Selektion der Fälle haben, die von den Krankenkassen geprüft werden. Der Einfluss der Krankenhäuser selbst wird hier gering sein. Dennoch sind Krankenhäuser gut beraten, die Korrektheit ihrer Abrechnungen und insbesondere die die Abrechnung stützende Dokumentation weiter zu optimieren. Dabei darf auch der Einfluss auf das Budget, die Wirtschaftsplanung und die Liquidität bei Prozessänderungen oder Änderungen des Abrechnungsverhaltens nicht aus den Augen verloren werden. Die finanziellen Auswirkungen des MDK-Reformgesetzes dürften jedoch schwer zu antizipieren sein. Krankenhäuser, die Leistungen erbringen, deren Indikation oder Durchführung unter stationären Bedingungen immer wieder strittig gestellt wird, sollten die vermehrte Klärung von Kostenübernahmen vor Leistungserbringung prüfen. Pauschale Vereinbarungen mit Krankenkassen mit einhergehendem Prüfverzicht sollen hingegen nicht mehr zulässig sein. Dafür soll bei Dissens über das MD-Gutachten eine einzelfallbezogene Erörterung zwischen Krankenhaus und Krankenkasse erfolgen, die Voraussetzung für eine nachfolgende gerichtliche Klärung des Sachverhaltes wird. Einwendungen und Tatsachen, die nicht im Rahmen dieser einzelfallbezogenen Erörterung vorgetragen wurden, sollen auch im nachfolgenden Gerichtsverfahren keine Rolle mehr spielen. Die näheren Einzelheiten sollen die Selbstverwaltungspartner bis zum 30. Juni 2020 festlegen. Bis dahin dürften wohl Verrechnungen der Krankenkassen und unter bestimmten Umständen auch noch Änderungen der Schlussrechnungen durch die Krankenhäuser zulässig bleiben. Perspektivisch ist jedoch zu erwarten, dass Krankenhäuser einmal gestellte Schlussrechnungen nur noch zur Umsetzung der Ergebnisse der MD-Gutachten oder rechtskräftiger Gerichtsurteile ändern dürfen. Auch deshalb müssen viele Krankenhäuser ihre Dokumentations-, Prüfund Abrechnungsprozesse nochmals deutlich verbessern. Kodierrevisionen nach ge- stellter Schlussrechnung dürften obsolet werden. Positiv ist anzumerken, dass der Gesetzgeber den Schlichtungsausschuss Bund reaktivieren will, dessen Aufgabe die verbindliche Klärung von Kodier- und Abrechnungsfragen von grundsätzlicher Bedeutung ist. Neben vielen andern Akteuren wird nun sogar einzelnen Krankenhäusern ein Anrufungsrecht zugestanden. Auch wenn der Zeitplan des Gesetzgebers zu ambitioniert erscheint, ist perspektivisch mit einem deutlichen Rückgang strittiger Kodier- und Abrechnungsfragen zu rechnen. Die Entscheidungen des Schlichtungsausschusses, die innerhalb von innerhalb von 8 Wochen nach Anrufung ergehen sollen, gelten als Kodierregeln und für Fälle, die ab dem 1. Tag des übernächsten auf die Entscheidung folgenden Monats aufgenommen werden, sowie „laufende Prüfungen“ der MD.

\section{Neue Strukturprüfungen durch den Medizinischen Dienst (MD)}

In der Hoffnung die Anzahl der Einzelfallprüfungen reduzieren zu können, sieht der Gesetzgeber zukünftig Strukturprüfungen durch den MD (ehemals MDK) vor ( $\S 275 \mathrm{~d}$ und 137 Abs. 3 SGB V). Krankenhäuser haben ab 2021 die Einhaltung von Strukturmerkmalen des OPS - vornehmlich vermutlich OPS-Komplexkodes - durch den MD begutachten zu lassen, bevor sie entsprechende Leistungen abrechnen. Grundlage der Strukturprüfungen wird eine vom MD Bund bis zum 30.04.2020 zu erlassende Richtlinie (§283 SGB V). Krankenhäuser erhalten bei Einhaltung der Strukturmerkmale eine Bescheinigung über das Ergebnis der Prüfung und die Dauer der Gültigkeit. Diese Bescheinigung ist bis spätestens zum 31.12.2020 an die Krankenkassen zu übermitteln, sonst dürfen entsprechende Leistungen ab dem Jahr 2021 nicht mehr vereinbart und auch nicht mehr abgerechnet werden ( 88 Abs. 4 KHEntgG). Nur wenn Krankenhäusern die Bescheinigung über die Einhaltung der Strukturmerkmale aus von ihnen nicht zu vertretenden Gründen erst nach dem 31.12.2020 vorliegt, oder Krankenhäuser gegen die Prüfergebnisse des MD klagen, dürfen Krankenhäuser bislang erbrachte Leistungen bis zum Abschluss der Strukturprüfung oder der Rechtskraft der gerichtlichen Entscheidung weiterhin vereinbaren und abrechnen. Krankenhäuser, die eines oder mehrere der nachgewiesenen Strukturmerkmale über einen Zeitraum von mehr als einem Monat nicht mehr einhalten (z. B. wegen Kündigung eines Facharztes oder Therapeuten), haben dies unverzüglich den Landesverbänden der Krankenkassen mitzuteilen. Die Folgen werden noch in der Richtlinie des MD Bund festgelegt. Einzelfallbezogene Prüfungen zur Einhaltung von Strukturmerkmalen des OPS sollen dafür zukünftig nicht mehr zulässig sein. Bislang ist nicht definiert, welche der Mindestkriterien der OPS-Kodes für die rheumatologische bzw. kinder- und jugendrheumatologische Komplexbehandlung als „Strukturkriterien" anzusehen sind. Wichtig ist, dass der MD nicht von sich aus die Strukturkriterien prüft, sondern Krankenhäuser ihn dazu explizit auffordern müssen. Rheumatologischen Kliniken sei deshalb dringlich angeraten, nach Veröffentlichung der Richtlinie im Jahr 2020 frühzeitig beim MD eine Strukturprüfung anzufordern, damit sie eine eventuell verspätete Prüfung nicht selbst zu verantworten haben. Erbringt ein Krankenhaus weitere erlösrelevante Komplexbehandlungen (z. B. Frührehabilitation, Geriatrische Komplexbehandlung, Schmerztherapie, Intensivmedizinische Komplexbehandlungen, Komplexbehandlungen des akuten Schlaganfalls, etc.) so müssen auch hier die Strukturprüfungen bis Ende 2020 abgeschlossen werden. Erfahrungen aus bisherigen Strukturprüfungen des MDK zeigen trotz guter Vorbereitung der Krankenhäuser hohe Durchfallquoten bei den ersten Prüfungen. Die damit verbundenen, potenziell dramatischen wirtschaftlichen Risiken sollten rheumatologische Kliniken nicht übersehen!

\section{Weitere Perspektiven}

Zum 1. Januar 2020 hätten die Selbstverwaltungspartner auf Bundesebene weitere pflegesensitive Bereiche in Krankenhäusern festlegen sollen, für die ab 2021 Pflegepersonaluntergrenzen gelten sollen. Vielfach wird gefordert, in diesem Schritt bereits alle internistischen Fachdisziplinen zu erfassen. Nachdem die Zahlen der DRG-Fallkostenkalkulation zeigen, dass in der Rheumatologie relativ wenig Pflegepersonal eingesetzt wird, könnten - in Abhängigkeit davon, wie die Untergrenzen ermittelt werden - viele rheumatologische Kliniken erheblich unter 
Druck geraten. Es empfiehlt sich, den Prozess der Ausweitung der Pflegepersonaluntergrenzen aufmerksam zu verfolgen.

Dass nach dem reformintensiven Jahr 2019 Ruhe in der Krankenhausfinanzierung eintreten wird, darf bezweifelt werden. Neben der mit dem MDK-Reformgesetz beschlossenen Erweiterung des Katalogs ambulanter Operationen und stationsersetzender Maßnahmen, der zukünftig auch eine Vielzahl nichtoperativer Leistungen enthalten soll, dürfte generell der Grenzbereich zwischen stationärer und ambulanter Versorgung in den Fokus rücken. Der Koalitionsvertrag sieht vor, dass eine Bund-Länder-Arbeitsgruppe bis zum Jahr 2020 Vorschläge für die Weiterentwicklung zu einer sektorenübergreifenden Versorgung vorlegt. Zusätzlich plant der Gesetzgeber eine umfassende Reform der Notfallversorgung. Die geplanten Maßnahmen haben das Potenzial, bisherige Strukturen fundamental zu verändern. Welche Fälle zukünftig von Krankenhäusern versorgt und ob diese dann nach einem stationären, ambulanten oder gänzlich neuen Abrechnungssystem vergütet werden, könnte einem erheblichen Wandel unterworfen werden. Aber auch das G-DRG-System selbst steht auf dem Prüfstand. Immer mehr Stimmen fordern eine Abkehr von der reinen Fallpauschalierung. Der „Pflexit“ und die Auswirkungen des MDK-Reformgesetzes zusam- men mit der stetig steigenden Komplexität erhöhen den Druck, Alternativen zu finden. Bundesgesundheitsminister Spahn hat bereits eine große Reform der Krankenhausfinanzierung für 2020 angekündigt. Wenn man ihn noch lässt ...

Prof. Dr. med. Heinz-Jürgen Lakomek, Geschäftsführer VRA

Prof. Dr. med. N. Roeder, Dr. med. Wolfgang Fiori

DRG-Research-Group, Roeder \& Partner

\section{KONTAKTADRESSE}

Verband Rheumatologischer Akutkliniken e. V.

Geschäftsstelle

Rechtsanwaltskanzlei Meyer-Koering

Schumannstr. 18, 10177 Berlin

Tel./Fax: 030/20 62 98-79/-82

E-Mail: gf@vraev.de

Internet: www.vraev.de

\section{IMPRESSUM}

\section{Verantwortlich für den Inhalt}

Prof. Dr. Heinz-Jürgen Lakomek, Direktor, Universitätsklinik für Geriatrie, Johannes Wesling Klinikum Minden 